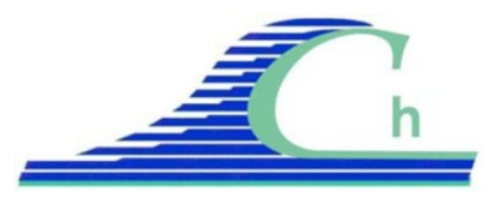

XII ${ }^{\text {ìmes }}$ Journées Nationales Génie Côtier - Génie Civil

Cherbourg, 12-14 juin 2012

DOI:10.5150/jngcgc.2012.094-D @ Editions Paralia CFL

disponible en ligne - http://www.paralia.fr - available online

\title{
Etats de la qualité des eaux en Algérie : cas des cours d'eau en région méditerranéenne (Kebir est, Seybouse et Medjerda), (extrême est algérien)
}

\author{
Larbi DJABRI ${ }^{1}$, Yassine FERRAH ${ }^{1}$, Saad BOUHSINA ${ }^{2}$, Fabrice CAZIER ${ }^{2}$, \\ Azzedine HANI ${ }^{1}$, Yacine DJABRI ${ }^{3}$, Antonio PULIDO BOSCH ${ }^{4}$, Habes SAMEH $^{4}$ \\ 1. Laboratoire Ressource en Eau et Développement Durable. Université Badji Mokhtar \\ Annaba, Algérie.Djabri_larbi@yahoo.fr \\ 2. Unité de Chimie Environnementale et Interactions sur le Vivant (UCEIV) EA 4492, \\ MREI 1 145, Avenue Maurice Schumann ULCO Dunkerque, France. \\ 3. Médecin Chef service Gynécologie, CHU, Annaba, Algérie. \\ 4. Université d’Almeria, Espagne.
}

\section{Résumé :}

Le présent travail traite de la qualité des eaux de quelques cours d'eau de l'extrême Est Algérien. Les Oueds (Seybouse, Medjerda, Kebir Est et Bouhamdene), reçoivent les eaux usées déversées par les localités et par les industries situées le long de ces cours d'eau. Ces eaux usées contribuent à la dégradation de la qualité des eaux des Oueds.

Notons que ces eaux sont utilisées pour l'irrigation, ce qui permet le déplacement des polluants vers les nappes superficielles soutenues par les Oueds.

Les analyses chimiques réalisées, ont montré des concentrations importantes en nutriments (nitrites, nitrates, ammonium et phosphore) et en métaux lourds (fer, zinc, manganèse, .....).

Les résultats obtenus, nous permettent de réaliser une étude comparative de la qualité des eaux des Oueds, on remarque que les eaux de l'Oued Bouhamdene, situé en zone de montagne sont moins polluées que les eaux de la Medjerda, une grande agglomération (Souk Ahras, avec 200000 habitants), reste le plus pollué. Les autres cours d'eau la Seybouse et le Kebir, présentent des pollutions intermittentes.

L'une des conséquences de cette pollution, reste l'eutrophisation de ces cours d'eau, conséquence du manque d'oxygène dans l'eau. Notons que toutes ces eaux des Oueds Seybouse Kebir Est, aboutissent en mer et peuvent entrainer un déséquilibre de l'environnement marin immédiat aux embouchures des Oueds. Les eaux de l'Oued Medjerda sont transfrontalières, elles s'écoulent vers la Tunisie.

Mots clés :

Oued - Echange de Bases - Gypsifères - Rejets - Thalweg - Algérie

\section{Introduction}

L’Algérie orientale est la région la plus arrosée du pays, elle reçoit annuellement une moyenne de précipitations allant de $300 \mathrm{~mm}$ à $1 \mathrm{~m}$ du sud vers le nord et atteint parfois 
1,5m en allant vers la méditerranée. Elle est aussi drainée par un réseau hydrographique très important avec une densité moyenne de drainage d'environ $2,11 \mathrm{~m} \mathrm{~km}^{2}$. Ces caractéristiques offrent à la région l'avantage d'être une ressource inépuisable en eau superficielle.

Le régime des cours d'eau diffère d'un bassin à un autre, il est fortement lié à un ensemble de facteurs physiques, géologiques ou encore climatiques qui régissent l'écoulement de l'eau superficielle. Vu les fortes disparités climatiques, topographiques et géologiques qui caractérisent l'ensemble des bassins versants du nord-est algérien, le régime de chaque bassin doit être étudié pour pouvoir faire la liaison entre l'écoulement fluvial et les différents facteurs cités précédemment.

\section{Situation géographique de la zone d'étude}

La zone d'étude est située à l'extrême nord-est algérien, entre les frontières algérotunisiennes et le bassin de Medjerda-Mellegue en Est, le bassin de Kebir- Rhumel en Ouest, la mer méditerranée au nord et les hauts plateaux Constantinois au sud (figure 1). Elle est de $14856 \mathrm{~km}^{2}$ de superficie, répartie entre le bassin de la Seybouse qui occupe une surface de $6071 \mathrm{~km}^{2}$, les bassins côtiers Constantinois du centre d'une superficie de $5582 \mathrm{~km}^{2}$ et les bassins côtiers Constantinois Est de $3203 \mathrm{~km}^{2}$ de surface.

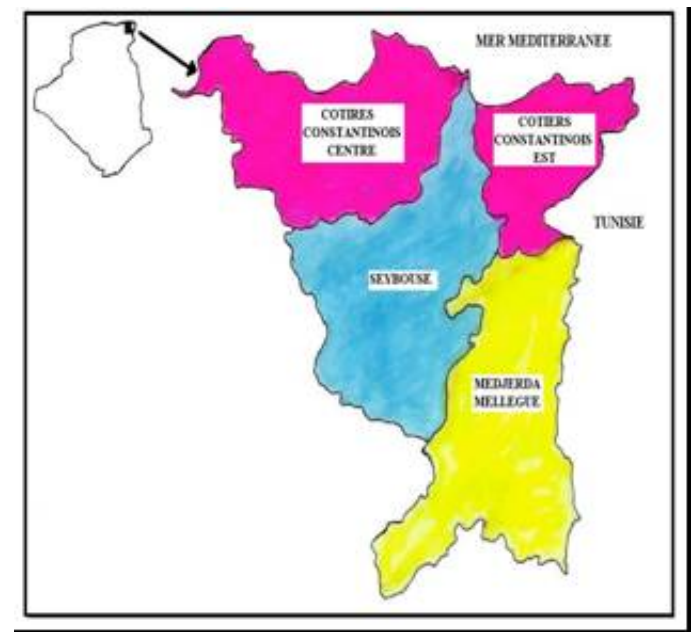

Figure 1. Situation géographique de la zone d'étude (BELHANACHI, 2003).

\section{Analyse des caractéristiques physiques des bassins versants de la région}

Les caractéristiques physiques des bassins influencent fortement leurs réponses hydrologiques et notamment le régime des écoulements en période de crue ou d'étiage. Le temps de concentration (Tc) qui caractérise en partie la vitesse et l'intensité de la réaction des bassins versants à une sollicitation des précipitations, est influencé par diverses caractéristiques morphologiques : en premier lieu, sa pente et son orientation. A ces facteurs s'ajoute la lithologie de terrain, le couvert végétal, et les caractéristiques 


\section{XII ${ }^{\text {èmes }}$ Journées Nationales Génie Côtier - Génie Civil \\ Cherbourg, 12-14 juin 2012}

du réseau hydrographique. La zone d'étude est formée d'un ensemble de bassins occupant une superficie totale de $8062 \mathrm{~km}^{2}$.

\section{Profils en long des principaux Thalwegs}

Le profil en long d'un cours d'eau traduit la variation de la cote du lit de l'Oued ; entre la source et l'exutoire ; en fonction de la distance. Il met en évidence les variations de pentes du lit de l'Oued. Les profils en long des cours d'eau principaux et leurs affluents dans les bassins de la Seybouse, Kebir Est et Medjerda sont représentés respectivement dans les figures 2 , 3, et 4 .

\subsection{Profil en long de l'Oued Seybouse}

Il s’étend de la zone semi-aride au Sud jusqu’à la mer méditerranée au Nord et est situé entre trois zones physiquement différentes :

- La haute Seybouse, caractérisée par des pentes relativement faibles et une lithologie à bonne perméabilité, favorisant l'infiltration.

- La moyenne Seybouse, située au nord de la première, caractérisant la région de Guelma. A ce stade, les pentes deviennent plus fortes, le drainage et le caractère torrentiel sont plus importants et la perméabilité diminue.

- La basse Seybouse, caractérise la zone littoral, à ce niveau les pentes redeviennent faibles, le terrain est perméables et la densité de drainage et le coefficient de torrentialité diminuent sauf pour les petits bassins de Oued Ressoul et Mellah où l'écoulement est relativement important.

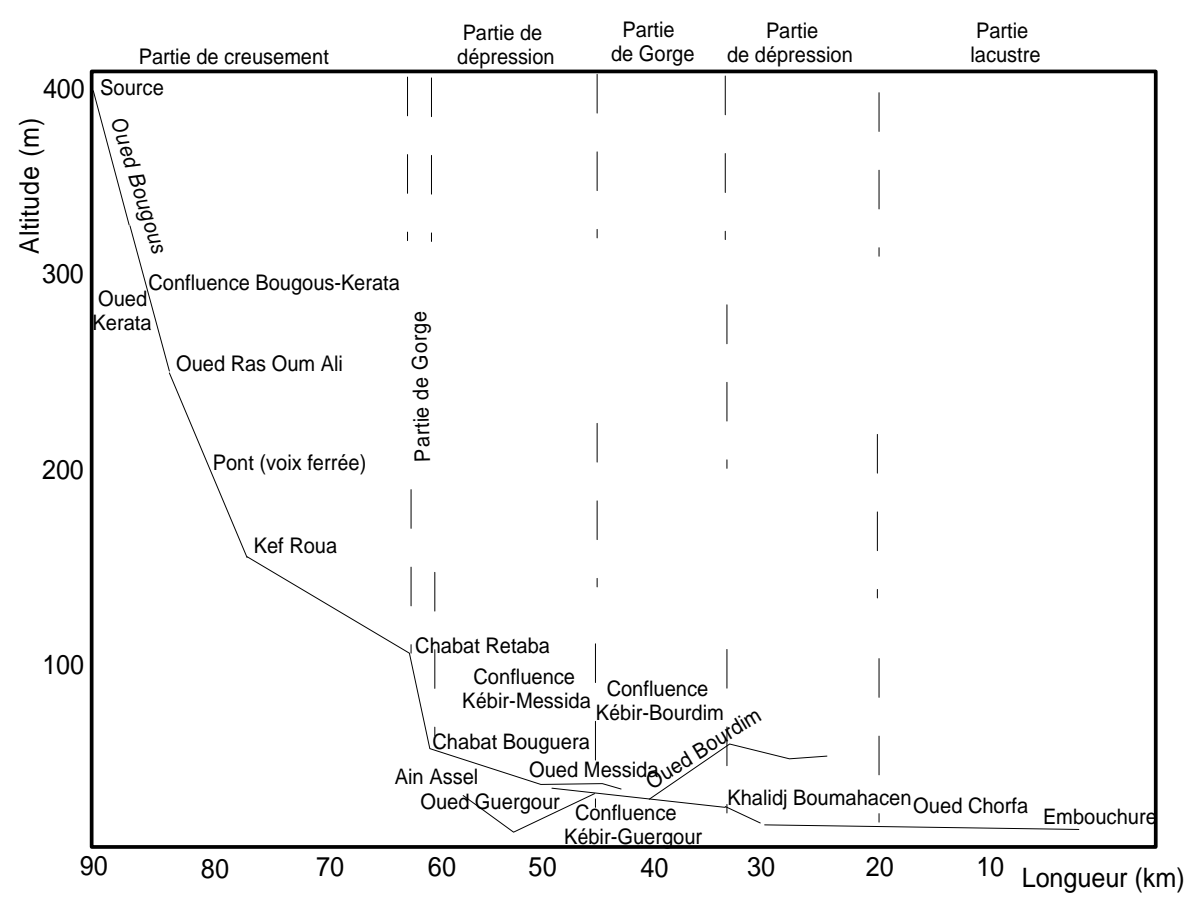

Figure 2. Profil en long de la Seybouse (DJABRI, 1996). 


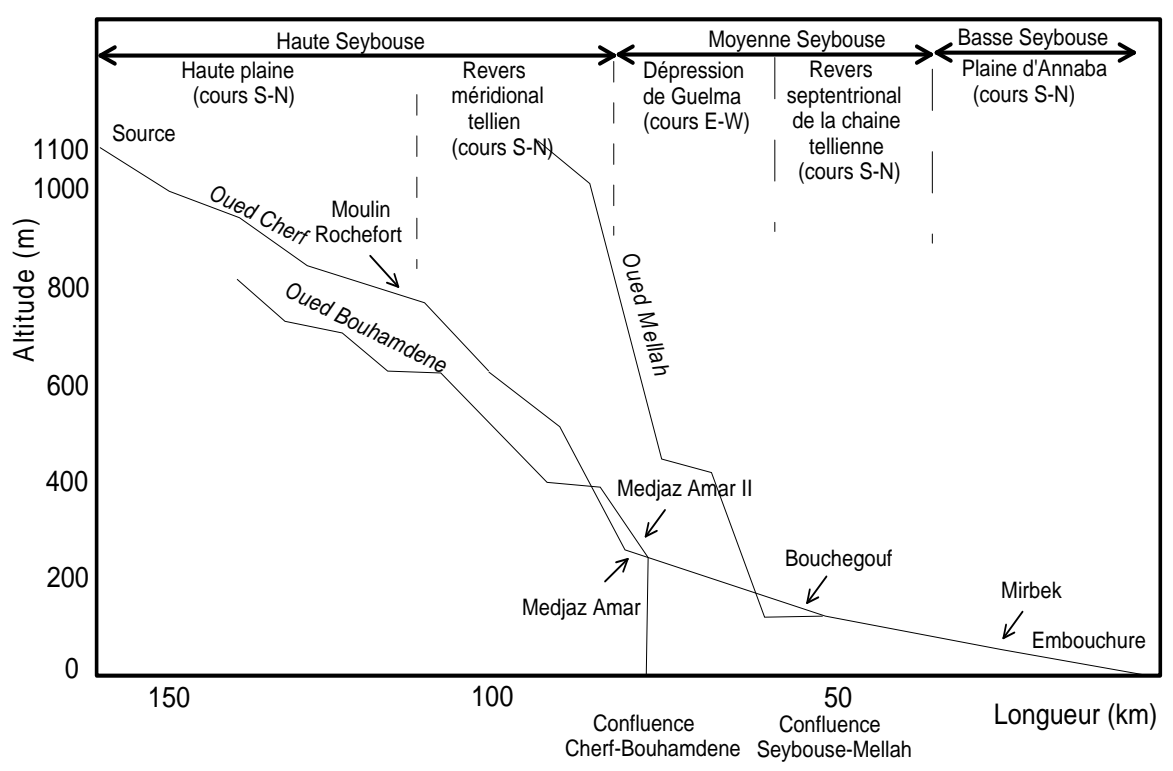

Figure 3. Profil en long de Kebir Est (BENCHAAR, 1999).

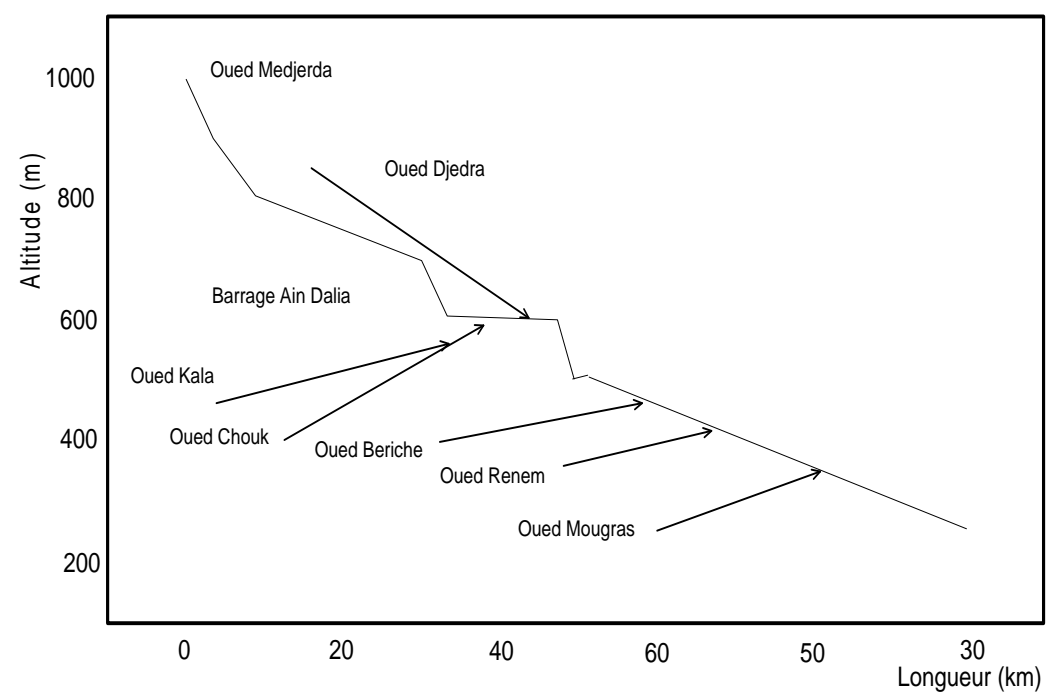

Figure 4. Profil en long de l'Oued Medjerda (GUASMI, 2005).

\subsection{Profil en long de l'Oued Kebir Est}

Se caractérise par une pente très forte dans sa partie amont. Ceci génère un écoulement important et torrentiel en période de pluie. Au niveau de la partie aval, la pente tend à devenir faible, entrainant une baisse des écoulements.

\subsection{Le profil en long de l’Oued Medjerda}

La dénivelée du profil en long est constante baisse. Cet Oued reçoit beaucoup d'eau en provenance de ses affluents, ce qui augmente les écoulements vers la Tunisie. L'Oued est entaillé dans une zone accidentée. 


\section{Qualité des eaux Transitant par ces Oueds}

L'observation du précédent tableau 2, montre que les axes F1-F2, sont les plus significatifs ils caractérisent les familles des eaux.

Tableau 2. L'analyse en composantes principales

\begin{tabular}{|c|c|c|c|c|c|c|}
\hline \multirow[b]{2}{*}{ Stations } & \multicolumn{6}{|l|}{ Paramètres } \\
\hline & Période & $F 1 \%$ & $F 2 \%$ & $F 1+F 2 \%^{\circ}$ & $\begin{array}{l}N \text { (nombre } \\
\text { d'analyses } \\
\text { utilisées) }\end{array}$ & $\begin{array}{l}R \\
\text { (coefficient } \\
\text { de } \\
\text { corrélation } \\
\text { seuil) }\end{array}$ \\
\hline Seybouse à Mirbek (in & Hautes eaux & 55.0 & 12.6 & 67.6 & 067 & 0.45 \\
\hline Djabri et all 2001) & Basses eaux & 39.6 & 26.0 & 65.6 & 024 & 0.69 \\
\hline Seybouse à Bouchegouf & Hautes eaux & 49.5 & 12.8 & 62.3 & 160 & 0.30 \\
\hline (in Djabri et all 200)1 & Basses eaux & 47.4 & 15.2 & 62.2 & 056 & 0.49 \\
\hline Seybouse à Medjez Amar & Hautes eaux & 47.4 & 13.7 & 61.1 & 153 & 0.31 \\
\hline (in Djabri et all 2001) & Basses eaux & 37.1 & 18.4 & 55.5 & 049 & 0.52 \\
\hline \multirow[t]{2}{*}{ Kebir Est à Ain Assel } & Hautes eaux & 51.8 & 14.0 & 65.8 & 126 & 0.34 \\
\hline & Basses eaux & 27.1 & 19.9 & 47.0 & 043 & 0.55 \\
\hline \multirow[t]{2}{*}{ Medjerda à Souk Ahras } & Hautes eaux & 38.0 & 21.0 & 60.0 & 098 & 0.30 \\
\hline & Basses eaux & 54.0 & 20.0 & 74.0 & 078 & 0.50 \\
\hline
\end{tabular}

\section{Détails de l'ACP, réalisée sur les eaux de l'Oued Medjerda (basses eaux)}

Le cercle formé par les axes F1-F2, (figure 5), donne plus de 78\% de l'information. L'observation du cercle, montre selon l'axe horizontal F1 une opposition entre les Eaux faiblement Minéralisées polluées par les nitrites (partie négative de l'axe F1) et les Eaux fortement Minéralisées partie positive de l'axe F1. Cette tendance serait liée au débit, qui entraîne une dilution des concentrations. Cependant on remarque que le calcium reste très influencé par les sulfates, les chlorures et les bicarbonates.

Selon l'axe vertical F2, on note une opposition entre les eaux bicarbonatées magnésiennes, chlorurées sodiques/calciques ou sulfatées sodiques/calciques polluées en nitrates (partie positive de l'axe) et les eaux polluées par NO2-, PO4, NH4+, DCO et DBO5 (partie négative de l'axe).

Les prélèvements réalisés aux points de rejets inventoriés au niveau de l'Oued Medjerda, nous ont permis de visualiser les variations de la qualité des eaux entre les différents points (figure 6). L'observation de la figure réalisée montre que les sulfates dominent pour tous les points, montrant ainsi l'influence des formations gypsifères sur la composition chimique des eaux. Ces sulfates s'accompagnent soit de calcium soit de 
sodium. Cette compétition est liée aux échanges de base se produisant entre l'eau et la roche. En effet aux cours de leur contact avec la roche les eaux s'enrichissent ou s'appauvrissent en éléments chimiques. Dans le cas présent l'Oued est entaillé dans des formations gypsifères qui en se dissolvant au contact de l'eau conduisent à une surminéralisation.

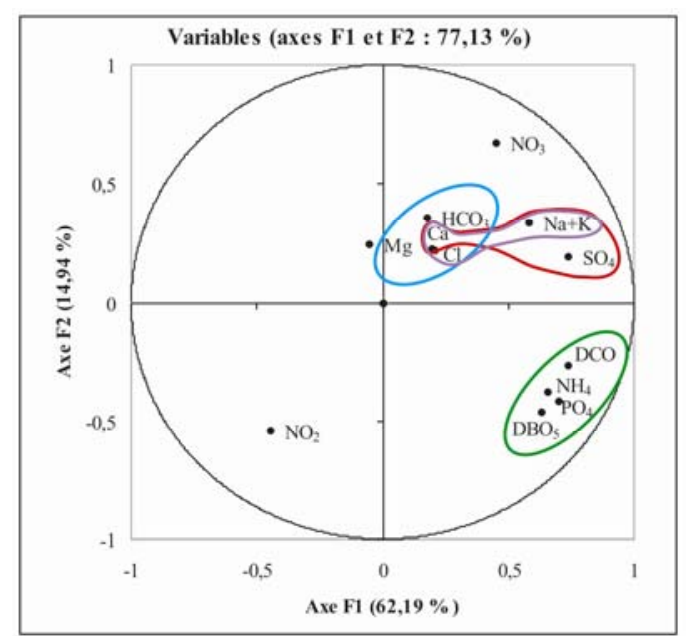

Figure 5. Cercle ACP, Oued Medjerda (GUASMI, 2009).

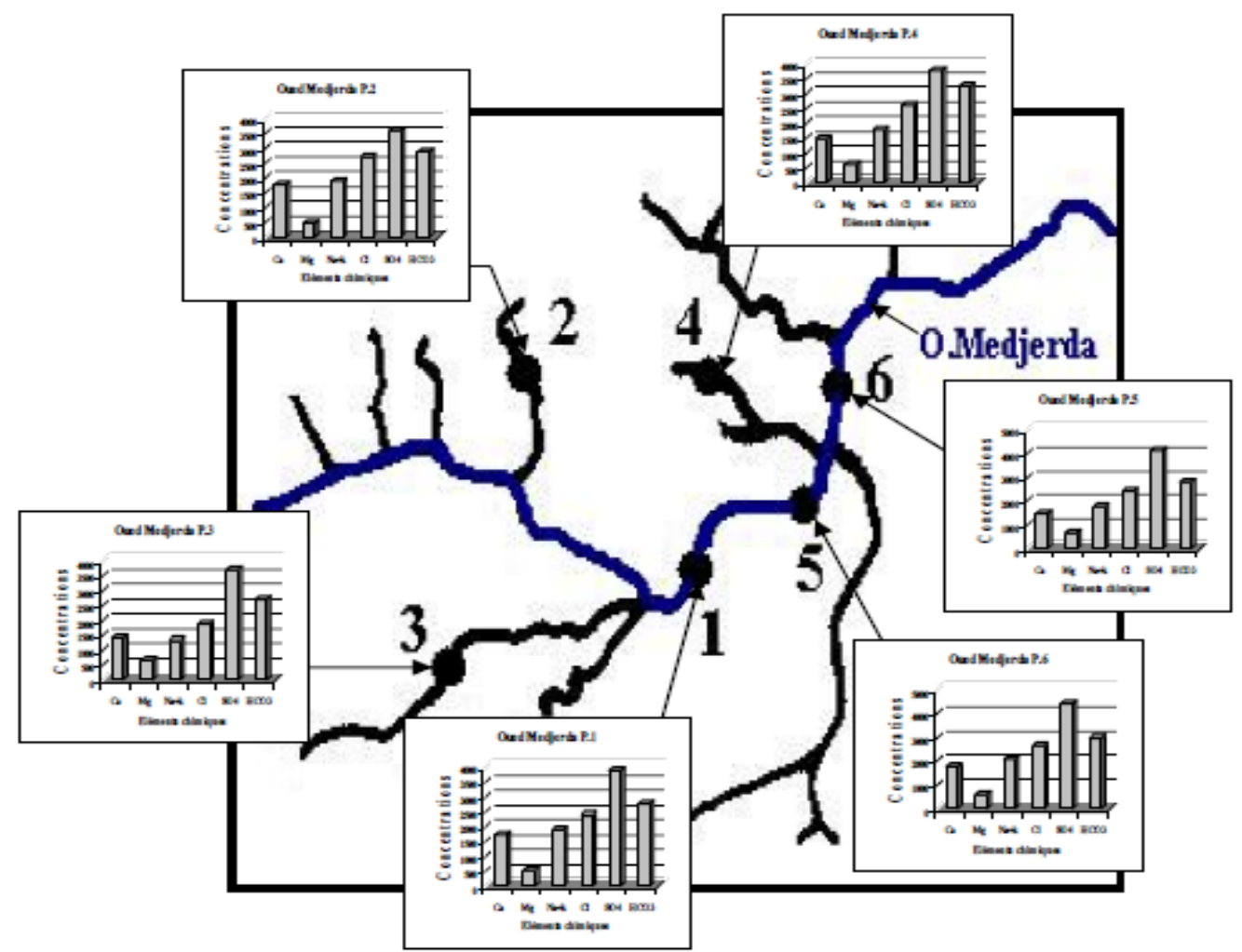

Figure 6. Variations de la composition chimique des eaux de l'Oued Medjerda. 


\section{XII ${ }^{\text {èmes }}$ Journées Nationales Génie Côtier - Génie Civil \\ Cherbourg, 12-14 juin 2012}

Notons que les résultats obtenus à partir de l'analyse en composantes principales, ont montré que la qualité des eaux des Oueds est particulièrement influencée par les formations géologiques à l'affleurement. Cependant les variations ponctuelles de la qualité des eaux sont à noter, ces dernières se produisent à l'approche des unités industrielles, des zones urbanisées ou lors des semences d'engrais. Le schéma suivant (figure 7), récapitule les variations de la composition chimique des eaux le long de l'Oued Seybouse. Nous remarquons que la qualité des eaux de l'Oued, reste conditionnée par les formations géologiques traversées par les eaux.

\section{Conclusion}

Le travail réalisé traite de la variation de la composition chimique des eaux des Oueds. Pour expliquer l'acquisition du chimisme des ces cours d'eau, nous avons utilisé plusieurs méthodes. Ainsi l'analyse en composantes principales, a montré une variation saisonnière de la composition chimique des eaux. Par ailleurs nous avons noté que la qualité des eaux dépend de l'activité anthropique et de la topographie et des affluents de chaque cours d'eau.

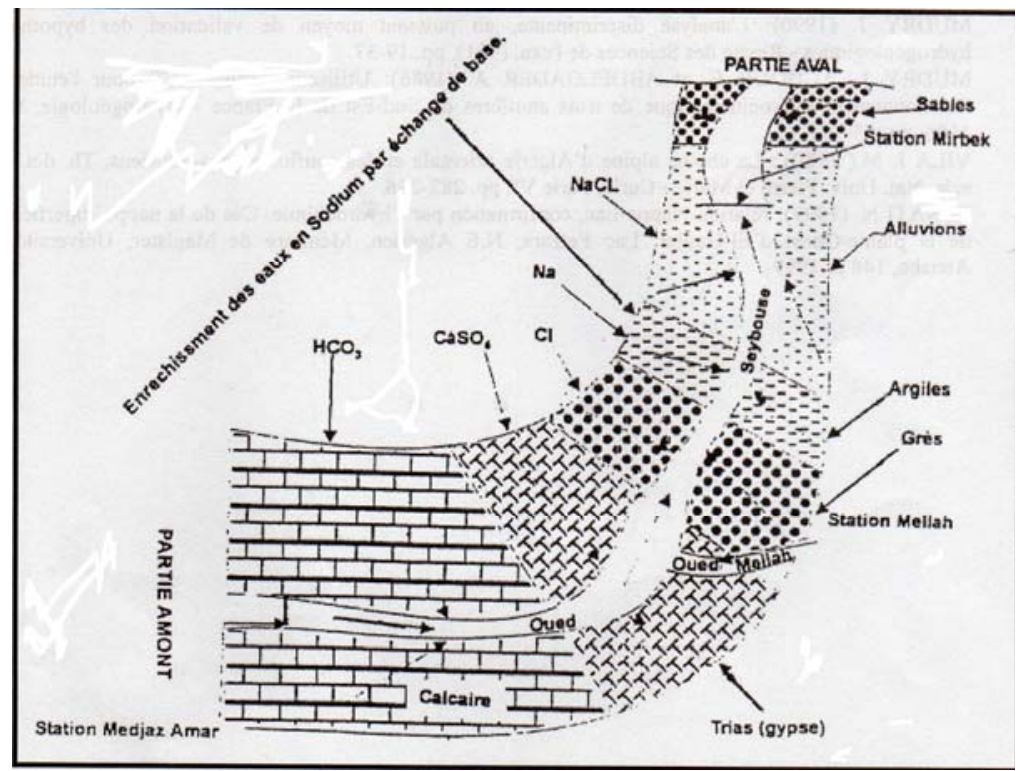

Figure 7. Mode d'acquisition du chimisme de l'Oued Seybouse (BELHANACHI, 2003).

\section{Références bibliographiques}

BELHANACHI A (2003). Etude hydrologique et hydrochimique dans les bassins versants du Nord-Est Algérien "Bassin de la Seybouse, Kébir Est, Kébir Ouest et Saf Saf". Mémoire de Magister, Université de Annaba.

BENCHAAR C (1999). Apport de l'analyse en composantes principales dans l'explication des mécanismes hydrochimiques des oueds Seybouse, Kébir Est et Kébir Ouest (Est Algérien). Mémoire de Magister. Université de Annaba, 177 p. 
Thème 6 - Gestion durable des zones littorales et estuariennes

DJABRI L. (1996). Mécanismes de la pollution et vulnérabilité des eaux de la Seybouse "Origine géologiques, industrielles, agricoles et urbaines". Thèse de doctorat. De l’Université d'Annaba. 278 p.

DJABRI L., HANI A., MESSADI D., MANIA J., MUDRY J. (2001). Mise en évidence du processus de salinité des eaux superficielles. Vérification par les ACP dans le secteur Annaba-Bouchegouf-Guelma. Tribune de l'eau, Vol. 54, nº 610/2, pp 29-43.

GUASMI I. (2005). Dégradation de la qualité de l'eau dans le bassin versant de l'oued Medjerda "Souk-Ahras". Mémoire de Magistère. Université de Batna.

GUASMI I. (2009). Pollution des eaux et pouvoir auto-épurateur de l'Oued Medjerda (Nord Est Algérien). Thèse de doctorat de l'université d'Annaba, 240 p. 